\title{
Selective inhibition of PCAF suppresses microglial-mediated $\beta$-amyloid neurotoxicity
}

\author{
SOO-YEON PARK ${ }^{1 *}$, YOO-HYUN LEE ${ }^{2 *}$, AH-REUM SEONG ${ }^{1}$, \\ JEONGMIN LEE ${ }^{3}$, WOOJIN JUN ${ }^{4}$ and HO-GEUN YOON ${ }^{1}$
}

\author{
${ }^{1}$ Department of Biochemistry and Molecular Biology, Center for Chronic Metabolic Disease Research, \\ Brain Korea 21 Project for Medical Sciences, Yonsei University College of Medicine, Seodaemun-gu, \\ Seoul 120-752; ${ }^{2}$ Department of Food Science and Nutrition, University of Suwon, Suwon, \\ Kyunggi-do 445-743; ${ }^{3}$ Department of Medical Nutrition, Kyung Hee University, Yongin-si, \\ Kyunggi-do 446-701; ${ }^{4}$ Department of Food and Nutrition, Chonnam National University, \\ Buk-Gu, Gwangju 500-757, Republic of Korea
}

Received March 6, 2013; Accepted May 22, 2013

DOI: 10.3892/ijmm.2013.1407

\begin{abstract}
Recent studies have emphasized the functional role of the P300/CBP-associated factor (PCAF) enzyme in resistance to $\beta$-amyloid $(A \beta)$-mediated neurotoxicity; however, the underlying mechanisms through which PCAF regulates inflammation and neurotoxicity have not yet been elucidated. In this study, we used computer-based molecular docking simulations to perform structure-based artificial screening for PCAF-specific inhibitors. Our results revealed that one of the compounds from the screened library, compound C-11, selectively inhibited PCAF, but not p300 or GCN5, with a half-maximal inhibitory concentration $\left(\mathrm{IC}_{50}\right)$ of approximately $0.25 \mu \mathrm{M}$. Furthermore, C-11 had no effects on the activities of other epigenetic enzymes. Western blot analysis using an antibody against acetyl-nuclear factor $-\kappa \mathrm{B}(\mathrm{NF}-\kappa \mathrm{B})$ demonstrated that PCAF mediated the $A \beta$-induced activation of $N F-\kappa B$ by acetylation at Lys-122. We also found that the knockdown of PCAF completely inhibited $\mathrm{A} \beta$-induced cytokine production in BV-2 cells in a similar manner to C-11 treatment. Finally, PCAF inhibition suppressed both A $\beta$-induced cytokine production and $\mathrm{A} \beta$-mediated neuronal cell death. Therefore, our results suggest that in neuronal cells, PCAF is a promising
\end{abstract}

Correspondence to: Dr Ho-Geun Yoon, Department of Biochemistry and Molecular Biology, Center for Chronic Metabolic Disease Research, Brain Korea 21 Project for Medical Sciences, Yonsei University College of Medicine, 134 Shinchon-dong, Seodaemun-gu, Seoul 120-752, Republic of Korea

E-mail: yhgeun@yuhs.ac

${ }^{*}$ Contributed equally

Key words: histone acetyltransferase, P300/CBP-associated factor, inflammation, nuclear factor- $\kappa \mathrm{B}$, neurotoxicity, $\beta$-amyloid, cytokine therapeutic target for alleviating the inflammatory progression of Alzheimer's disease.

\section{Introduction}

Chronic inflammation is associated with the pathogenesis of several human diseases, including cancer and neurodegenerative diseases $(1,2)$. Nuclear factor $-\kappa \mathrm{B}(\mathrm{NF}-\kappa \mathrm{B})$ is the most well-known regulator of inflammatory signaling (3), and activated $\mathrm{NF}-\kappa \mathrm{B}$ is commonly observed in inflammatory lesions during pathogenesis (4). Evidence to date demonstrates that the functions of $\mathrm{NF}-\kappa \mathrm{B}$ are modulated by diverse posttranscriptional modifications (5). Among these modifications, acetylation has been shown to enhance the nuclear localization of $N F-\kappa B$ and lead to the transcription of $N F-\kappa B$ target genes $(6,7)$. NF- $\kappa \mathrm{B}$ is mainly acetylated by $\mathrm{p} 300 / \mathrm{CBP}$ and p300/CBP-associated factor (PCAF) (8). p300/CBP acetylates multiple lysine residues of $\mathrm{NF}-\kappa \mathrm{B}$, including Lys-122, -123 , $-218,-221$ and -310 , activating its transcriptional activity (7). On the other hand, PCAF specifically acetylates only Lys-122 of NF- $\mathrm{BB}$ (9). The difference between acetylation by PCAF and p300 on the regulation of $\mathrm{NF}-\kappa \mathrm{B}$ function, however, is largely unknown. Conversely, the blocking of $\mathrm{NF}-\kappa \mathrm{B}$ acetylation by histone acetyl transferase (HAT) inhibitors diminishes the nuclear retention and transcriptional activity of NF- $\kappa \mathrm{B}(10-12)$. Thus, the pharmacological inhibition of HAT activity may be a useful method for the treatment of chronic inflammation.

Neuroinflammation is a hallmark of neurodegenerative diseases that is linked to glial cell activation (13-15). The abnormal activation of microglia promotes neuronal injury through the release of pro-inflammatory and cytotoxic factors, including tumor necrosis factor- $\alpha$ (TNF- $\alpha$ ), interleukin (IL)-1 $\beta$, inducible nitric oxide synthase (iNOS), cyclooxygenase-2 (COX-2), nitric oxide and reactive oxygen species that contribute to localized and more widespread central nervous system injuries (16-18). NF- $\mathrm{B}$ is also involved in neuroinflammation and neuronal cell death induced by $\beta$-amyloid (A $\beta$ ) (19-21). Consistent with this, the immunoreactivity of the 
RelA (p65) subunit of NF- $\kappa \mathrm{B}$ in the brains of patients with Alzheimer's disease is stronger in the neurons, astrocytes and microglial cells surrounding amyloid plaques (22). In a recent study, we reported the inhibitory effects of gallic acid, a HAT inhibitor, on NF- $\mathrm{NB}$ activity and the activation of microglial inflammation (10). In addition, we have previously shown that gallic acid inhibits multiple HAT enzymes, including p300 and PCAF (23). Of note, it has recently been demonstrated that PCAF knockout mice develop resistance to amyloid toxicity and have altered memory capacities, suggesting the importance of the PCAF enzyme in the development of neurodegenerative diseases $(24,25)$. However, the detailed mechanisms through which PCAF promotes $\mathrm{A} \beta$-induced microglial inflammation and neurotoxicity remain unclear.

In this study, we used computer-based molecular docking simulations to screen for PCAF-specific inhibitors. We demonstrate that the inhibition of PCAF abrogates lipopolysaccharide (LPS)-induced NF- $\kappa \mathrm{B}$ activation and the acetylation of $\mathrm{NF}-\kappa \mathrm{B}$ at Lys-122. Finally, we used co-culture analysis, demonstrating that a PCAF inhibitor efficiently prevented neuronal cell death caused by $A \beta$-induced neurotoxicity. Collectively, our results demonstrate that the selective inhibition of NF- $\kappa \mathrm{B}$ acetylation by a PCAF inhibitor shows promise for the treatment of neurodegenerative diseases.

\section{Materials and methods}

Molecular docking analysis and chemicals. Structure-based artificial screening with computer-based molecular docking simulations was conducted by InnoPharmaScreen Inc. (Asan, Korea). The X-ray crystal structure of the HAT domain of human PCAF bound to coenzyme A (CoA; Protein Data Bank ID: 1CM0) was used for the initial structure of the target protein. CoA was removed and atomic hydrogens were added to produce a complete molecular model of the target protein for use in the molecular docking simulations. Docking simulations were performed between the target model and the ChemBridge chemical library that consists of 450,000 unique compounds. Binding efficiency was based on the binding force between the target molecule and the test compound as calculated by the Gold 4.0.1 software package (Cambridge Crystallographic Data Centre, Cambridge, UK). The top 10 docking positions for each compound were calculated, and the library search efficiency was $100 \%$.

Cell culture, reagents and antibodies. Murine BV-2 cells and Neuro-2A cells were obtained from the American Type Culture Collection (ATCC; Manassas, VA, USA; CRL no. 2270). Fetal bovine serum (FBS), trypsin-EDTA, penicillin and streptomycin were purchased from Gibco-BRL (Gaithersburg, MD, USA). 3-(4,5-Dimethylthiazol-2-ly)-2,5diphenyl tetrazolium bromide (MTT) was purchased from Sigma-Aldrich (St. Louis, MO, USA). A $\beta_{1-42}$ was purchased from Bachem (Bubendorf, Switzerland). Other chemicals used were purchased from Sigma-Aldrich. BV-2 cells were cultured in Dulbecco's modified Eagle's medium (Gibco-BRL) containing 5\% heat-inactivated endotoxin-free FBS, $2 \mathrm{mM}$ glutamine, $100 \mu \mathrm{g} / \mathrm{ml}$ streptomycin and $100 \mathrm{U} / \mathrm{ml}$ penicillin in a humidified $5 \% \mathrm{CO}_{2}$ atmosphere at $37^{\circ} \mathrm{C}$. Neuro-2A cells were cultured in modified Eagle's medium (Gibco-BRL) containing
$10 \%$ heat-inactivated endotoxin-free FBS, 2 mM glutamine, $100 \mu \mathrm{g} / \mathrm{ml}$ streptomycin, and $100 \mathrm{U} / \mathrm{ml}$ penicillin in a humidified $5 \% \mathrm{CO}_{2}$ atmosphere at $37^{\circ} \mathrm{C}$. A $\beta$ peptides were dissolved in phosphate-buffered saline (PBS) and pre-incubated at $37^{\circ} \mathrm{C}$ for 5 days to allow for fibril formation. The peptides were stored at $-20^{\circ} \mathrm{C}$ until use. Compound $\mathrm{C}-11$ was dissolved in dimethyl sulfoxide (DMSO) and later diluted with distilled water (the final concentration of DMSO was $<0.5 \%$ ). For the co-culture experiments, $\mathrm{BV}-2$ cells were treated with various concentrations of $\mathrm{C}-11(0.1-1.0 \mu \mathrm{M}$ final concentration) for $12 \mathrm{~h}$ prior to stimulation with $5 \mu \mathrm{M}$ aggregated $\mathrm{A} \beta_{1-42}$ (the $\mathrm{A} \beta+\mathrm{C}-11$ group), $5 \mu \mathrm{M}$ non-aggregated $\mathrm{A} \beta_{1-42}$, or medium only (control) for $24 \mathrm{~h}$. Conditioned medium (CM) from the BV-2 cells and primary microglial cells was collected, centrifuged and transferred to the Neuro-2A cells for a further $24 \mathrm{~h}$. CM from the mediumonly treated cells was used as the control. Following incubation, cell viability was measured by MTT assay and western blot analysis was performed.

HAT, histone deacetylase (HDAC), histone methyltransferase (HMT) and sirtuin 1 (SIRT1) activity assays. HeLa cell nuclear extract was prepared as previously described (26). HAT and HDAC activity assays were performed with nuclear extracts using commercially available kits according to the manufacturer's instructions (BioVision, Inc., Milpitas, CA, USA). For in vitro HAT activity assays, recombinant HAT proteins were incubated with HAT assay buffer [50 mM HEPES pH 8.0, 10\% glycerol, $1 \mathrm{mM}$ DTT, $1 \mathrm{mM}$ phenylmethanesulfonyl fluoride (PMSF), $10 \mathrm{mM}$ sodium butyrate and $1 \mu \mathrm{l}\left[{ }^{3} \mathrm{H}\right]$-acetyl-CoA], $5 \mu \mathrm{g}$ histone $\mathrm{H} 4$ tail peptides, and/or indicated concentration of inhibitors at $30^{\circ} \mathrm{C}$ for $1 \mathrm{~h}$. Samples were separated on a $15 \%$ sodium dodecyl sulfate-polyacrylamide gel electrophoresis (SDS-PAGE) gel and analyzed by autoradiography. HMT activity assays were performed using the assay buffer and core histones from the HMT Assay Reagent kit (Upstate Biotechnology, Inc., Lake Placid, NY, USA) according to the manufacturer's instructions. Core histones were incubated for $1 \mathrm{~h}$ at $30^{\circ} \mathrm{C}$ in methyltransferase buffer (final concentration $50 \mathrm{mM}$ Tris pH 8.0, $1 \mathrm{mM}$ PMSF, and 0.5 mM DTT $), 1 \mu \mathrm{l}(0.55 \mu \mathrm{Ci})$ S-adenosyl-L-[methyl- $\left.{ }^{3} \mathrm{H}\right]$-methionine (Amersham Pharmacia Biotech, Inc., Piscataway, NJ, USA) and $10 \mu \mathrm{g} / \mathrm{ml}$ HeLa nuclear extract. The total volume of the reaction mixture was $30 \mu 1$. The reaction was spotted on $\mathrm{P}-81$ paper for scintillation counting. The P-81 paper was washed 3 times with $10 \%$ trichloroacetic acid (Upstate Biotechnology, Inc.) for $15 \mathrm{~min}$, washed with $95 \%$ ethanol for $5 \mathrm{~min}$ at room temperature and allowed to dry. Dry P-81 papers were counted with a multi-purpose LS 6500 scintillation counter (Beckman Coulter, Indianapolis, IN, USA). SIRT1 activity was assayed using a SIRT1/Sir2 Deacetylase Fluorometric assay kit (CycLex Co., Ltd., Nagano, Japan) according to the manufacturer's instructions.

Lentiviral shRNAs. For silencing PCAF expression, 2 pairs of oligonucleotides that encoded PCAF-specific shRNA were purchased from MISSION shRNA (Sigma-Aldrich). Lentiviral particles were prepared using pLKO.1-PURO PCAF shRNA via a 3 plasmid co-transfection according to the instructions of the manufacturer (Invitrogen, Carlsbad, CA, USA). The BV-2 cells were transfected with the lentivirus. After 3 days of incubation, the lentivirus from the culture medium was collected 
and concentrated in a Centricon-plus-20 centrifugal filter device (Millipore, Billerica, MA, USA). Lentivirus PURO shRNA was generated as the control.

Cell viability assay (MTT assay). Cell viability was measured to determine the cytotoxicity of the $\mathrm{A} \beta$ peptides on neuronal cells. Neuro-2A cells were seeded at $1 \times 10^{4}$ to $1 \times 10^{5}$ cells in a 96-well plate. Following $12 \mathrm{~h}$ of incubation, the medium was replaced with $\mathrm{CM}$ from the BV-2 cell cultures treated with or without $A \beta$ and different concentrations of $C-11$. After $24 \mathrm{~h}$, the $\mathrm{CM}$ was replaced with non-CM, $15 \mu \mathrm{l}$ MTT solution ( $2 \mathrm{mg} / \mathrm{ml}$ final concentration) was added for $90 \mathrm{~min}$ at $37^{\circ} \mathrm{C}$, and the absorbance was recorded at $570 \mathrm{~nm}$. A reference was recorded at $630 \mathrm{~nm}$ using a microplate reader (Model 550; Bio-Rad, Hercules, CA, USA).

Western blot analysis. The treated cells were washed with cold PBS, scraped off the culture dishes and harvested. The cells were incubated for $20 \mathrm{~min}$ in lysis buffer containing $0.5 \%$ Triton X-100, 20 mM HEPES (pH 7.4), $150 \mathrm{mM} \mathrm{NaCl}$, $2 \mathrm{mM}$ DTT and $1 \mathrm{mM}$ PMSF. The lysates were centrifuged at $20,000 \times \mathrm{g}$ for $10 \mathrm{~min}$ at $4^{\circ} \mathrm{C}$. The protein concentrations in the clarified lysates were determined with the Bradford assay using bovine serum albumin as a reference. Total cell lysate proteins were separated on 8 or $12 \%$ SDS-PAGE gels and transferred onto nitrocellulose membranes. The membranes were blocked by incubating for $12 \mathrm{~h}$ in $5 \%(\mathrm{w} / \mathrm{v})$ non-fat $\mathrm{Difco}^{\mathrm{TM}}$ skim milk blocking buffer. The blocked membranes were incubated overnight at $4^{\circ} \mathrm{C}$ with primary antibodies that recognize iNOS (1:1,000 dilution), COX-2 (1:1,000), IL-1 $\beta$ (1:500), $\mathrm{NF}-\kappa \mathrm{B}(\mathrm{p} 65 ; 1: 500)$ or $\beta$-actin $(1: 5,000)$. The antibody against acetyl-NF- $\kappa \mathrm{B}$ (K122) was raised against the synthetic peptide, CIHSFQNLGIQCV $\mathrm{AC}_{\mathrm{C}}$ KKRDLE, by GenScript (Piscataway, NJ, USA). After washing 3 times with PBS and 0.1\% Tween-20, the membranes were incubated with secondary horseradish peroxidase-conjugated antibody $(1: 1,000)$ for $1 \mathrm{~h}$. The bands were detected using the enhanced chemiluminescence system (Amersham Pharmacia Biotech, Inc.) according to the manufacturer's instructions.

RNA extraction and real-time PCR analysis. Total RNA from the Neuro-2A, BV-2 and primary microglial cells was extracted using TRIzol reagent (Invitrogen) according to the manufacturer's instructions. The mRNA levels of iNOS, COX-2 and IL-1 $\beta$ were determined by real-time PCR (ABI PRISM 500 Sequence Detection System, Applied Biosystems, San Jose, CA, USA). cDNA amplification was performed in duplicate in 20- $\mu 1$ reaction mixtures containing 2 X SYBR-Green Master Mix (Roche, Indianapolis, IN, USA) and $10 \mathrm{pM}$ forward and reverse primers. The initial denaturation step was $5 \mathrm{~min}$ at $95^{\circ} \mathrm{C}$ followed by 40 amplification cycles of $30 \mathrm{sec}$ at $95^{\circ} \mathrm{C}$, $30 \mathrm{sec}$ at $58^{\circ} \mathrm{C}$ and $30 \mathrm{sec}$ at $72^{\circ} \mathrm{C}$ with a final $10 \mathrm{~min}$ extension at $72^{\circ} \mathrm{C}$. The results were analyzed using $\mathrm{ABI}$ sequence detector software version 2.3. Relative mRNA expression of the target genes was calculated after normalizing to GAPDH expression and was expressed as the fold induction. The primers used for amplification were as follows: human IL-1 $\beta$ gene forward, 5'-GTTGACGGACCCCAAAAGAT-3' and reverse, 5'-AAGG TCCCACGGGAAAGACAC-3'; human COX-2 gene forward, 5'-GAGTGGGAGGCACTTGCATT-3' and reverse, 5'-TGGA
Table I. Gold fitness score for each compound.

\begin{tabular}{rrrrrrrr}
\hline No. & Score & No. & Score & No. & Score & No. & Score \\
\hline C-1 & 85.55 & C-26 & 73.43 & C-51 & 72.12 & C-76 & 70.86 \\
C-2 & 84.11 & C-27 & 73.42 & C-52 & 71.94 & C-77 & 70.81 \\
C-3 & 81.48 & C-28 & 73.33 & C-53 & 71.89 & C-78 & 70.77 \\
C-4 & 81.00 & C-29 & 73.29 & C-54 & 71.87 & C-79 & 70.65 \\
C-5 & 80.75 & C-30 & 73.18 & C-55 & 71.76 & C-80 & 70.58 \\
C-6 & 80.58 & C-31 & 73.14 & C-56 & 71.69 & C-81 & 70.53 \\
C-7 & 80.21 & C-32 & 73.10 & C-57 & 71.59 & C-82 & 70.42 \\
C-8 & 77.79 & C-33 & 73.05 & C-58 & 71.51 & C-83 & 70.40 \\
C-9 & 77.56 & C-34 & 72.97 & C-59 & 71.51 & C-84 & 70.39 \\
C-10 & 77.45 & C-35 & 72.95 & C-60 & 71.38 & C-85 & 70.32 \\
C-11 & 76.83 & C-36 & 72.92 & C-61 & 71.37 & C-86 & 70.28 \\
C-12 & 76.35 & C-37 & 72.90 & C-62 & 71.36 & C-87 & 70.28 \\
C-13 & 76.27 & C-38 & 72.84 & C-63 & 71.36 & C-88 & 70.20 \\
C-14 & 75.87 & C-39 & 72.83 & C-64 & 71.36 & C-89 & 70.20 \\
C-15 & 75.42 & C-40 & 72.80 & C-65 & 71.33 & C-90 & 70.19 \\
C-16 & 74.93 & C-41 & 72.64 & C-66 & 71.30 & C-91 & 70.16 \\
C-17 & 74.73 & C-42 & 72.55 & C-67 & 71.28 & C-92 & 70.15 \\
C-18 & 74.33 & C-43 & 72.46 & C-68 & 71.15 & C-93 & 70.07 \\
C-19 & 74.20 & C-44 & 72.42 & C-69 & 71.09 & C-94 & 70.04 \\
C-20 & 74.10 & C-45 & 72.37 & C-70 & 71.06 & C-95 & 69.98 \\
C-21 & 74.04 & C-46 & 72.36 & C-71 & 71.03 & C-96 & 69.94 \\
C-23 & 73.91 & C-47 & 72.30 & C-72 & 71.00 & C-97 & 69.94 \\
C-25 & 73.47 & C-48 & 72.21 & C-73 & 71.00 & C-98 & 69.93 \\
\hline & C-49 & 72.19 & C-74 & 70.98 & C-99 & 69.92 \\
C-50 & 72.16 & C-75 & 70.86 & C-100 & 69.84 \\
\hline
\end{tabular}

GGCGAAGTGGGTTTTA-3'; human iNOS gene forward, 5'-TCTTGGAGCGAGTTGTGGAT-3' and reverse, 5'-GGGT GGTAATGTCCAGGAAGT-3'; and human GAPDH gene forward, 5'-GTGTTCCTACCCCCAATGTGT-3' and reverse, 5'-AGGAGACAACCTGGTCCTCAGT-3'. All reactions were performed in triplicate. Relative expression levels and standard deviations (SDs) were calculated using the comparative value method.

\section{Results}

Structure-based artificial screening of PCAF-specific inhibitors. To develop PCAF-specific inhibitors, we performed structure-based artificial screening using computer-based molecular docking simulations (Fig. 1A). We initially selected 50 compounds with a binding force of at least 70 after the molecular docking simulation (Table I). Among these, compound number 11 (C-11) displayed the most significant inhibitory effect against the PCAF enzyme (Fig. 1B). Furthermore, C-11 selectively inhibited PCAF, but not p300 and GCN5 (Fig. 1C). Thus, we selected $\mathrm{C}-11$ as the putative PCAF inhibitor for our experiments.

To examine the enzyme specificity of $\mathrm{C}-11$, we examined whether C-11 inhibits other epigenetic enzymes, including HDAC, HMT and SIRT1. As shown in Fig. 2A, C-11 failed to 


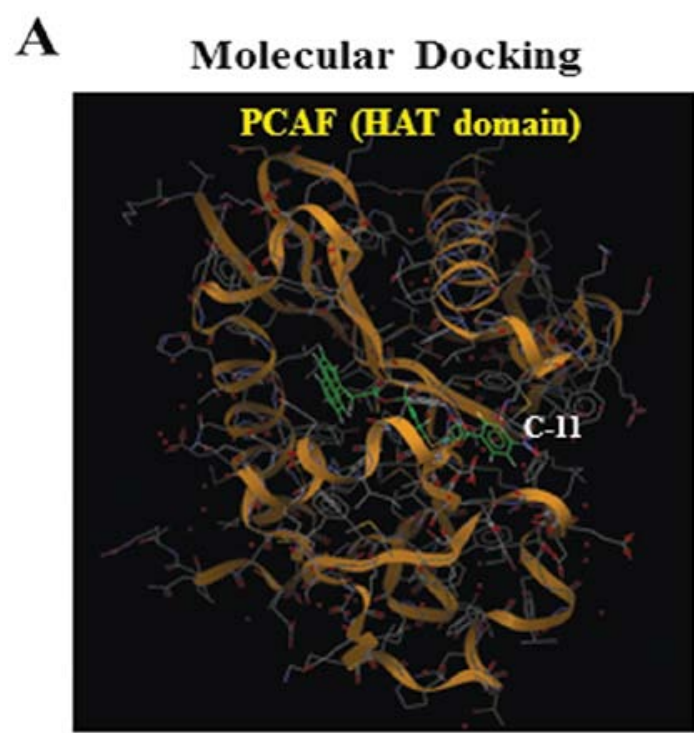

B

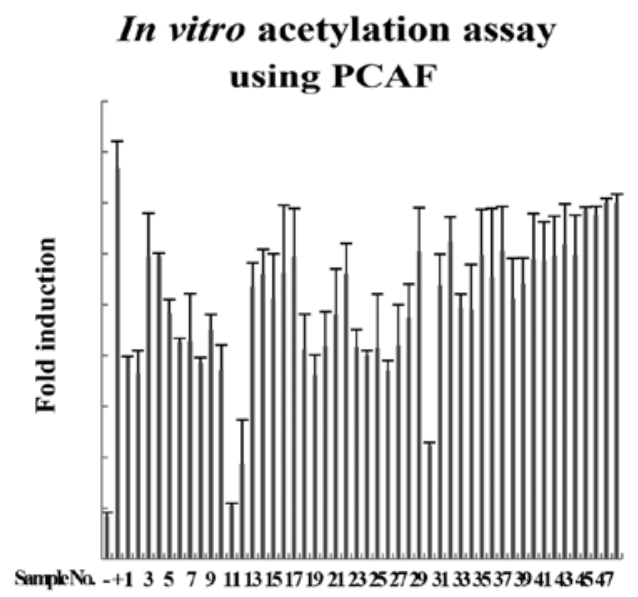

C

\section{In vitro acetylation assay}

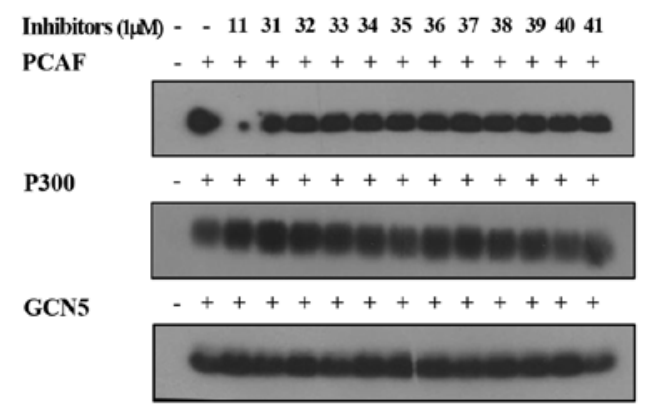

Figure 1. Identification of P300/CBP-associated factor (PCAF) inhibitors by structure-based virtual screening. (A) Molecular docking analysis was performed between the 450,000 compounds of the ChemBridge chemical library and the molecular model of PCAF. The Gold 4.0.1 software package was used to score the binding forces between molecules. (B) C-11 possesses the highest anti-PCAF activity among the putative PCAF inhibitors. Histone acetyl transferase (HAT) activity was assayed with a colorimetric assay kit with recombinant PCAF proteins as the source of HAT enzymes. The results are shown as the means \pm SD of 3 independent experiments. (C) C-11 selectively inhibits PCAF activity. In vitro HAT activity assays were performed with the indicated HAT enzymes in the presence of chemical compounds $(1 \mu \mathrm{M})$ and a synthetic histone 4 tail peptide. inhibit the enzyme activities of HDAC and SIRT1. Furthermore, C-11 had no effect on HMT activity (Fig. 2B). These results demonstrate that C-11 specifically inhibits PCAF acetyltransferase activity but not the activity of other epigenetic enzymes. As several HAT inhibitors (HATi) have recently been shown to possess anti-PCAF activity $(12,23,27)$, we compared the relative anti-PCAF activity of C-11 with other HATi. As shown in Fig. 2C, C-11 had the highest anti-PCAF activity among the reported HATi with a half-maximal inhibitory concentration of approximately $0.25 \mu \mathrm{M}$ (Fig. 2C and D).

Inhibition of PCAF suppresses $A \beta$-induced $N F-\kappa B$ acetylation at Lys-122. Since PCAF was found to activate NF- $\mathrm{KB}$-mediated transcription in response to cytokines (6), we first examined whether the inhibition of PCAF by $\mathrm{C}-11$ suppresses $\mathrm{NF}-\kappa \mathrm{B}$ activity by inhibiting the acetylation of NF- $\kappa \mathrm{B}$. Since PCAF is known to acetylate NF- $\kappa B$ at Lys-122 (6), we first generated an acetyl-specific NF- $\mathrm{BB}$ antibody that recognizes acetylated NF- $\mathrm{KB}$ at Lys-122. Polyclonal antibodies were generated against the NF- $\mathrm{KB}$ acetyl-peptide, ${ }^{109} \mathrm{CIHSFQNLGIQCV}_{\mathrm{AC}} \mathrm{KKRDLE}^{127}$, and the antisera were examined by western blot analysis following in vitro acetylation reactions with recombinant PCAF enzyme and GST-p65 substrate (Fig. 3A, upper panel). A peptide competition assay clearly demonstrated the specificity of the NF- $\mathrm{kB}$ Lys(Ac)-122 antibody, as the complete blocking of NF- $\mathrm{KB}$ acetylation was observed by the antibody raised against the acetyl-peptide, but not by an antibody raised against a non-acetyl-peptide (Fig. 3A, lower panel). In addition, non-acetyl-NF-кB antibody failed to detect the acetylation that resulted from an in vitro HAT reaction and thus confirmed the specificity of the acetylspecific NF- $\kappa \mathrm{B}$ antibody for the PCAF-mediated acetylation of NF- $\kappa$ B at Lys-122 (Fig. 3A, lower panel).

We then examined whether $A \beta$ treatment induces NF- $\kappa B$ activation through the acetylation of NF- $\mathrm{KB}$ at Lys-122. A $\beta$ treatment efficiently induced the activation of $\mathrm{NF}-\kappa \mathrm{B}$, and this was inhibited by the knockdown of PCAF (Fig. 3B and C). Importantly, $A \beta$ treatment also increased the acetylation of NF- $\kappa B$ at Lys-122, which was verified by 2 antibodies that recognize acetylated NF- $\kappa$ B at Lys-122 (Fig. 3D). The acetylation of NF- $\kappa B$ at Lys-122 was specifically mediated by PCAF, as shRNA against PCAF, but not control shRNA, abrogated the A $\beta$-induced acetylation of NF- $\mathrm{kB}$. Collectively, these data suggest that PCAF mediates the $A \beta$-induced activation of $\mathrm{NF}-\kappa \mathrm{B}$ through the acetylation of NF- $\mathrm{KB}$ at Lys-122.

Pharmacological inhibition of PCAF abrogates the $A \beta$-induced activation of $N F-\kappa B$. We then investigated the inhibitory effects of PCAF on NF- $\mathrm{KB}$ activity and cytokine production. We first assessed the effect of knocking down PCAF on cytokine production by BV-2 cells. Consistent the results from our previous study (10), A $\beta$ treatment significantly increased the expression of cytokine genes in BV-2 cells (Fig. 4A). Of note, treatment with shRNA against PCAF markedly diminished the $A \beta$-induced cytokine production in BV-2 cells when compared with control shRNA (Fig. 4A). Importantly, PCAF inhibition by $\mathrm{C}-11$ markedly reduced the $\mathrm{A} \beta$-induced cytokine production in BV-2 cells. Furthermore, we also observed similar results with LPS treatment (Fig. 4B). These data collectively demonstrate that the inhibition of 

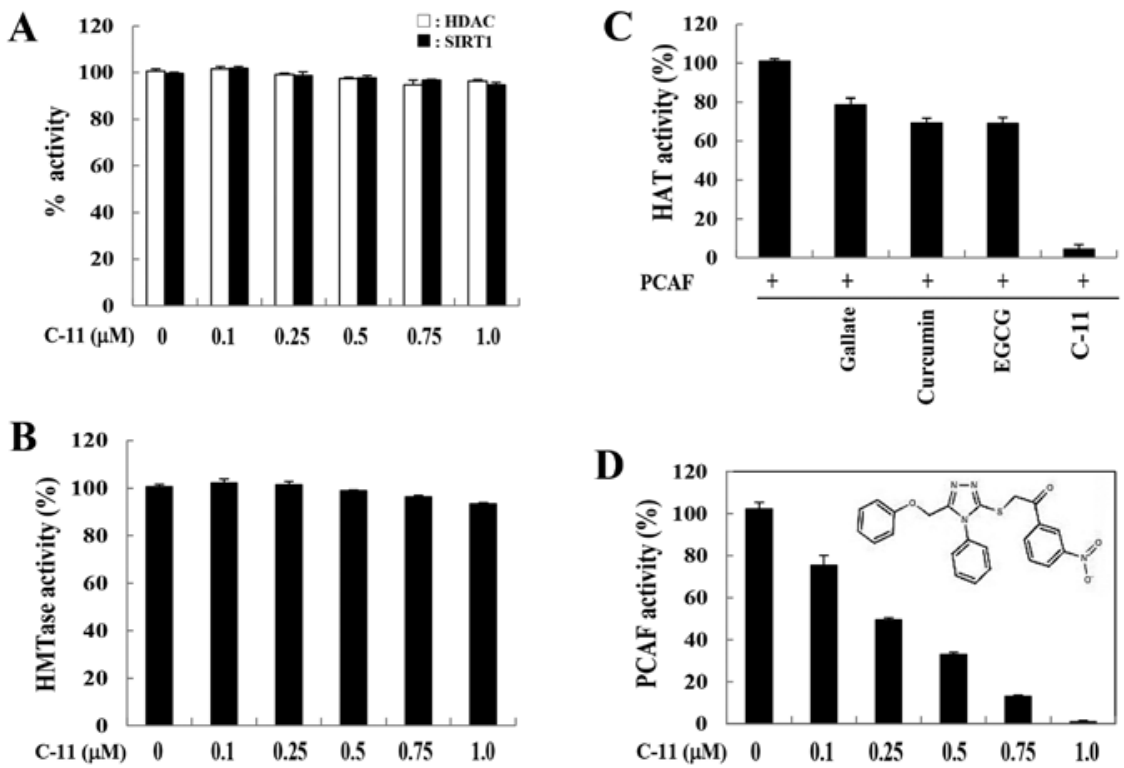

Figure 2. Compound C-11 selectively inhibits P300/CBP-associated factor (PCAF) activity. (A) C-11 treatment had no effect on histone deacetylase (HDAC) activity as assayed with a colorimetric assay kit. Sirtuin 1 (SIRT1) activity was assayed with a SIRT1/Sir2 deacetylase fluorometric assay kit. Deacetylase enzyme activity was assessed by measuring the change in fluorescence intensity. The results represent the means \pm SD of 3 independent experiments. (B) Histone methyltransferase (HMT) filter-binding assays were performed in 30- $\mu 1$ reaction volumes in the presence or absence of C-11 with HeLa cell nuclear extracts as the enzyme source. (C) C-11 has potent anti-PCAF activity. PCAF activity was assayed with a histone acetyl transferase (HAT) activity colorimetric assay kit with recombinant PCAF as the source of HAT enzymes. The results represent the means \pm SD of 3 independent experiments. (D) C-11 inhibits PCAF activity in a dose-dependent manner. PCAF activity was assessed with the indicated concentrations of C-11.

A

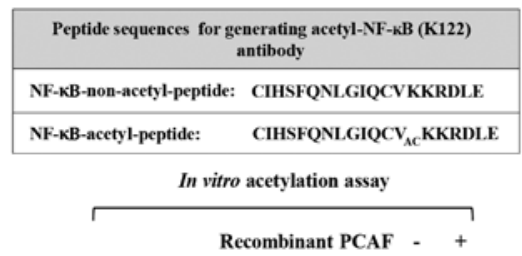

$\$$

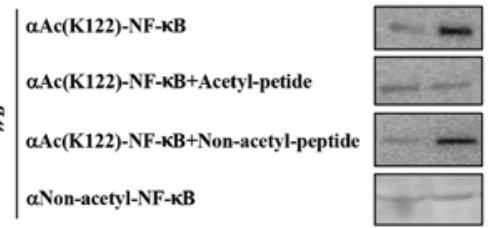

C

\section{Reporter assay (NF-кB-Luc)}

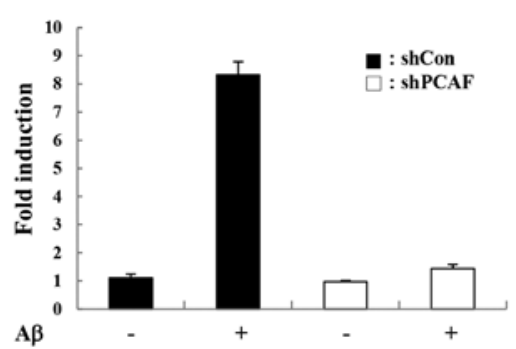

B

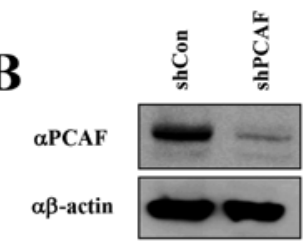

$\alpha \beta$-actin

D

BV2 cell

$\mathbf{A} \beta$

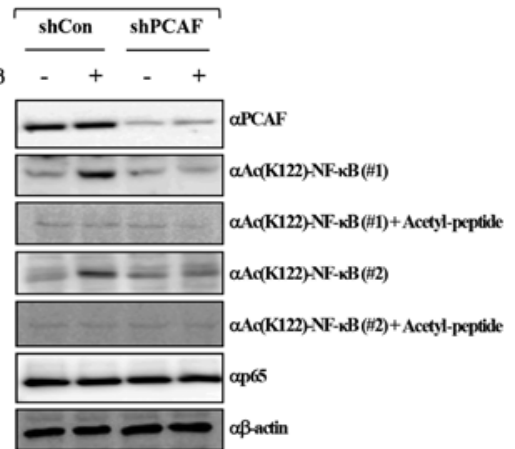

Figure 3. C-11 inhibits the P300/CBP-associated factor (PCAF)-mediated acetylation of NF- $\mathrm{kB}$ on Lys-122. (A) Peptide sequences for the generation of the acetylation-specific NF- $\mathrm{\kappa B}$ antibody. In vitro acetylation assays were performed with recombinant PCAF enzyme and the indicated GST-p65 proteins. Western blot analysis was performed with acetyl-NF- $\mathrm{kB}$ (K122) antibodies and/or $1 \mu \mathrm{g} / \mathrm{ml}$ of non-acetyl-peptide or acetyl-peptide. (B) Validation of the effect of shRNA against PCAF. Western blot analysis was performed with the indicated antibodies. BV-2 cells were transfected with lentiviral shRNA against PCAF, and lentivirus PURO shRNA was generated as the control. (C) Knockdown of PCAF reduced A $\beta$-induced NF- $\mathrm{BB}$ activity in BV-2 cells. BV-2 cells were transfected with an NF- $\mathrm{KB}$ binding site-driven luciferase reporter plasmid and treated with $1 \mu \mathrm{M} \mathrm{A} \beta$. Whole cell extracts were used in the luciferase assay, and the results are presented as the means of 2 independent experiments performed in triplicate. (D) Knockdown of PCAF prevented A $\beta$-induced p65 acetylation at Lys-122 in vivo. BV-2 cells were treated with lentiviral shRNA against PCAF and/or A $\beta$. The level of p65 acetylation was assayed by western blot analysis using an antibody against acetylated p65 at Lys-122. 
A

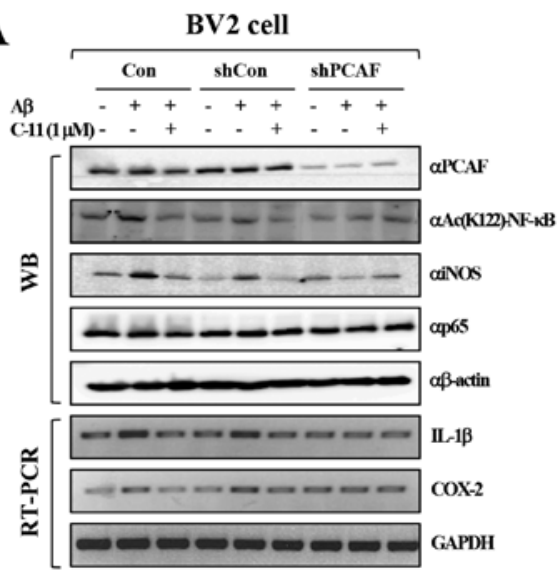

$\mathbf{C}$

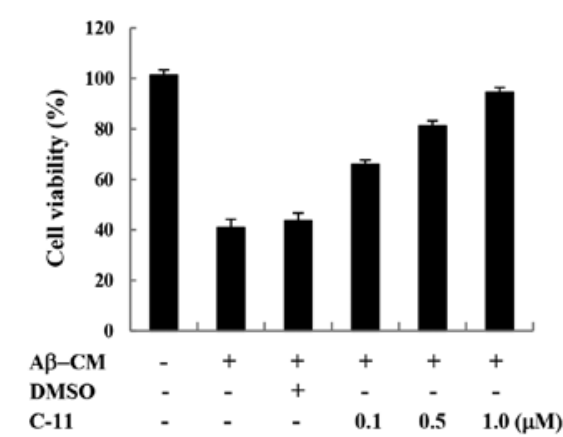

B

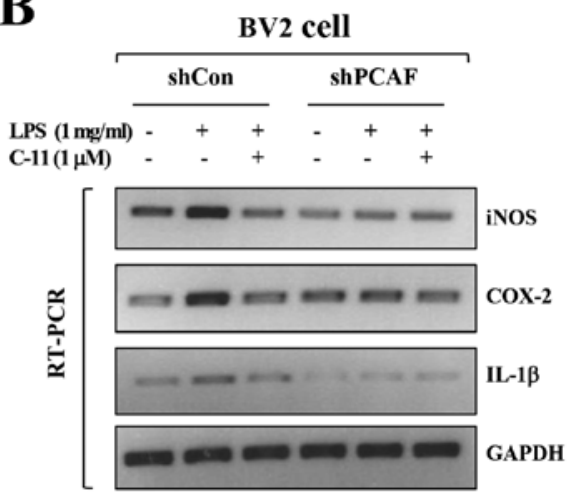

qRT-PCR

D

Co-culture assay (Neuro-2A)

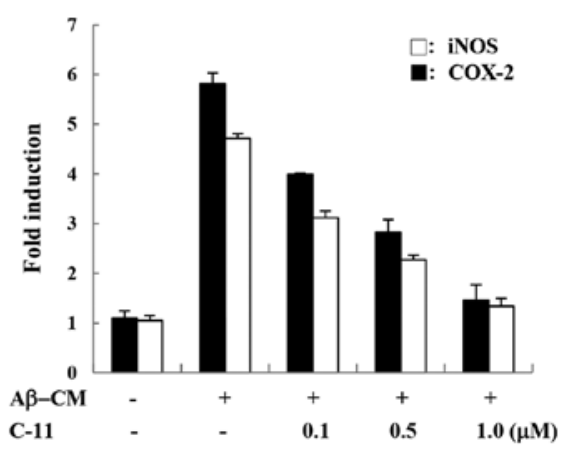

Figure 4. Selective inhibition of P300/CBP-associated factor (PCAF) abolishes microglial-mediated cytokine production and A $\beta$-mediated neurotoxicity. (A) PCAF inhibition suppresses the A $\beta$-induced expression of cytokine genes. BV-2 cells were transfected with shRNA against PCAF and after $48 \mathrm{~h}$ the cells were treated with $A \beta$ and/or $C-11$. The protein levels were assayed by western blot analysis (WB) using the indicated antibodies. (B) PCAF inhibition prevented LPS-induced cytokine production. Total RNA was prepared from each sample and used in RT-PCR reactions to measure the expression of the NF- $\mathrm{kB}$ target genes. (C) Treatment with a PCAF inhibitor prevented A $\beta$-induced neuronal cell death. The conditioned medium from BV-2 microglial cells was transferred to the Neuro-2A cells, and cell viability was assessed with the MTT assay. (D) Effect of a PCAF inhibitor on the expression of cytokines in Neuro-2A cells was analyzed with real-time PCR. Columns represent the averages of 3 independent experiments and error bars represent the standard deviations.

PCAF abolishes NF- $\mathrm{NB}$-mediated cytokine production by blocking the acetylation of $\mathrm{NF}-\kappa \mathrm{B}$ at Lys-122.

Inhibition of PCAF prevents A $\beta$-induced neuronal cell death. Since PCAF inhibition suppresses the expression of pro-inflammatory cytokines, we examined the neuroprotective effects of PCAF inhibition on BV- 2 microglial-mediated A $\beta$ neurotoxicity. Consistent with the results from our previous study showing the dose-dependent cytotoxicity of gallic acid in the same system (10), treatment with A $\beta$-conditioned medium (CM) from BV-2 microglial cells reduced the viability of Neuro-2A cells, and $\mathrm{C}-11$ reversed the $\mathrm{A} \beta-\mathrm{CM}-$ mediated neuronal cell death in a dose-dependent manner (Fig. 4C). Real-time PCR demonstrated that $\mathrm{C}-11$ consistently suppressed the $\mathrm{A} \beta-\mathrm{CM}$-induced cytokine production in Neuro-2A cells (Fig. 4D). Collectively, these data demonstrate that the inhibition of PCAF by $\mathrm{C}-11$ inhibits $\mathrm{NF}-\kappa \mathrm{B}$ acetylation, which in turn suppresses $A \beta$-induced neuroinflammation and $\mathrm{A} \beta$-mediated neurotoxicity.

\section{Discussion}

In this study, we demonstrated a role for PCAF in microglial inflammation through acetylation-dependent $\mathrm{NF}-\kappa \mathrm{B}$ activa- tion, and provide a rationale for the use of PCAF as a promising therapeutic target for the treatment of Alzheimer's disease. Previous studies have shown that PCAF acetylates NF- $\kappa B$ at Lys-122 in response to cytokine signaling and that this in turn, leads to increased NF- $\kappa \mathrm{B}$ activity (8). However, due to the unavailability of an acetyl-specific NF- $\mathrm{B}$ antibody, it is still unclear as to whether PCAF indeed acetylates NF- $\kappa$ B at Lys-122. In the current study, we utilized an acetyl (Lys-122)specific $\mathrm{NF}-\kappa \mathrm{B}$ antibody, clearly demonstrating that PCAF mediates the $A \beta$-induced activation of $N F-\kappa B$ through the acetylation of $\mathrm{NF}-\kappa \mathrm{B}$ at Lys-122. Furthermore, we did not observe any change in the acetylation of NF- $\kappa \mathrm{B}$ at Lys-122 after knocking down p300 (data not shown). Thus, our study confirms the specific acetylation of NF- $\kappa \mathrm{B}$ at Lys-122 by PCAF.

Accumulating evidence suggests that microglial inflammation by cytokines aggravates $A \beta$-induced neurotoxicity $(28,29)$. Furthermore, we have previously demonstrated that the inhibition of $\mathrm{NF}-\kappa \mathrm{B}$ acetylation with a HAT inhibitor, such as gallate significantly reduces $A \beta$-mediated neuronal cell death through the suppression of inflammatory signaling (10). The pharmacological inhibition of NF- $\kappa \mathrm{B}$ acetylation may, therefore, prove useful as a therapeutic intervention in Alzheimer's disease. In this context, a previous studies have demonstrated that PCAF 
knockout mice are insensitive to $A \beta$ neurotoxicity $(24,25)$, suggesting a pivotal role for PCAF in the regulation of $A \beta$-induced neurotoxicity and the memory capacity of the brain. In support of these findings, to our knowledge, in this study, we demonstrate for the first time that the pharmacological inhibition of PCAF prevents A $\beta$-induced cytokine production and toxicity in neuronal cells. The most promising compound selected by our molecular docking analysis was compound C-11, a PCAF-specific inhibitor. C-11 treatment specifically inhibited the cytokine-induced activation of $\mathrm{NF}-\kappa \mathrm{B}$ by preventing the acetylation of $\mathrm{NF}-\kappa \mathrm{B}$ at Lys-122. Importantly, C-11 had no effect on the activity of any of the other epigenetic enzymes examined. More importantly, the knockdown of PCAF with shRNA had a similar effect on cytokine production and the viability of neuronal cells as did C-11 treatment.

In conclusion, our data suggest that the selective inhibition of NF- $\kappa \mathrm{B}$ acetylation by PCAF inhibition is a possible therapeutic approach for alleviating the inflammatory progression of Alzheimer's disease.

\section{Acknowledgements}

This study was supported by a grant from the Korea Health Care Technology R\&D Project, Ministry for Health, Welfare and Family Affairs, Republic of Korea (no. A092039).

\section{References}

1. Ferguson LR and Laing WA: Chronic inflammation, mutation and human disease. Mutat Res 690: 1-2, 2010.

2. Dinarello CA: Inflammation in human disease: anticytokine therapy. Biol Blood Marrow Transplant 15 (Suppl 1): 134-136, 2009.

3. Hayden MS and Ghosh S: Signaling to NF-kappaB. Genes Dev 18: 2195-2224, 2004.

4. Aradhya S and Nelson DL: NF-kappaB signaling and human disease. Curr Opin Genet Dev 11: 300-306, 2001.

5. Campbell KJ and Perkins ND: Post-translational modification of RelA (p65) NF-kappaB. Biochem Soc Trans 32: 1087-1089, 2004.

6. Chen LF, Mu Y and Greene WC: Acetylation of RelA at discrete sites regulates distinct nuclear functions of NF-kappaB. EMBO J 21: 6539-6548, 2002

7. Chen LF, Fischle W, Verdin E and Greene WC: Duration of nuclear NF-kappaB action regulated by reversible acetylation. Science 293: 1653-1657, 2001.

8. Chen LF and Greene WC: Shaping the nuclear action of NF-kappaB. Nat Rev Mol Cell Biol 5: 392-401, 2004.

9. Greene WC and Chen LF: Regulation of NF-kappaB action by reversible acetylation. Novartis Found Symp 259: 208-217, 2004.

10. Kim MJ, Seong AR, Yoo JY, et al: Gallic acid, a histone acetyltransferase inhibitor, suppresses beta-amyloid neurotoxicity by inhibiting microglial-mediated neuroinflammation. Mol Nutr Food Res 55: 1798-1808, 2011.

11. Seong AR, Yoo JY, Choi K, et al: Delphinidin, a specific inhibitor of histone acetyltransferase, suppresses inflammatory signaling via prevention of $\mathrm{NF}-\kappa \mathrm{B}$ acetylation in fibroblast-like synoviocyte MH7A cells. Biochem Biophys Res Commun 410 581-586, 2011.
12. Choi KC, Jung MG, Lee $\mathrm{YH}$, et al: Epigallocatechin-3-gallate, a histone acetyltransferase inhibitor, inhibits EBV-induced B lymphocyte transformation via suppression of RelA acetylation. Cancer Res 69: 583-592, 2009.

13. Holmes C: Systemic inflammation and Alzheimer's Disease. Neuropathol Appl Neurobiol. 39: 51-68, 2012.

14. Cameron B and Landreth GE: Inflammation, microglia, and Alzheimer's disease. Neurobiol Dis 37: 503-509, 2010.

15. Jones RW: Inflammation and Alzheimer's disease. Lancet 358: 436-437, 2001.

16. Ekdahl CT, Kokaia Z and Lindvall O: Brain inflammation and adult neurogenesis: the dual role of microglia. Neuroscience 158: 1021-1029, 2009.

17. Kim YS and Joh TH: Microglia, major player in the brain inflammation: their roles in the pathogenesis of Parkinson's disease. Exp Mol Med 38: 333-347, 2006.

18. Min KJ, Yang MS, Kim SU, Jou I and Joe EH: Astrocytes induce hemeoxygenase-1 expression in microglia: a feasible mechanism for preventing excessive brain inflammation. J Neurosci 26: $1880-1887,2006$

19. Jayasooriya RG, Kang CH, Seo MJ, Choi YH, Jeong YK and Kim GY: Exopolysaccharide of Laetiporus sulphureus var. miniatus downregulates LPS-induced production of NO, PGE(2), and TNF-alpha in BV2 microglia cells via suppression of the NF-kappaB pathway. Food Chem Toxicol 49: 2758-2764, 2011.

20. Ha SK, Moon E and Kim SY: Chrysin suppresses LPS-stimulated proinflammatory responses by blocking NF-kappaB and JNK activations in microglia cells. Neurosci Lett 485: 143-147, 2010.

21. Wilms H, Rosenstiel P, Sievers J, Deuschl G, Zecca L and Lucius R: Activation of microglia by human neuromelanin is NF-kappaB dependent and involves p38 mitogen-activated protein kinase: implications for Parkinson's disease. FASEB J 17: 500-502, 2003

22. Kaltschmidt B, Uherek M, Volk B, Baeuerle PA and Kaltschmidt C: Transcription factor NF-kappaB is activated in primary neurons by amyloid beta peptides and in neurons surrounding early plaques from patients with Alzheimer disease. Proc Natl Acad Sci USA 94: 2642-2647, 1997.

23. Choi KC, Lee YH, Jung MG, et al: Gallic acid suppresses lipopolysaccharide-induced nuclear factor-kappaB signaling by preventing RelA acetylation in A549 lung cancer cells. Mol Cancer Res 7: 2011-2021, 2009.

24. Duclot F, Jacquet C, Gongora C and Maurice T: Alteration of working memory but not in anxiety or stress response in p300/CBP associated factor (PCAF) histone acetylase knockout mice bred on a C57BL/6 background. Neurosci Lett 475: 179-183, 2010.

25. Maurice T, Duclot F, Meunier J, et al: Altered memory capacities and response to stress in $\mathrm{p} 300 / \mathrm{CBP}$-associated factor (PCAF) histone acetylase knockout mice. Neuropsychopharmacology 33: 1584-1602, 2008.

26. Yoon HG, Chan DW, Huang ZQ, et al: Purification and functional characterization of the human $\mathrm{N}-\mathrm{CoR}$ complex: the roles of HDAC3, TBL1 and TBLR1. EMBO J 22: 1336-1346, 2003.

27. Kusio-Kobialka M, Dudka-Ruszkowska W, Ghizzoni M, et al: Inhibition of PCAF by anacardic acid derivative leads to apoptosis and breaks resistance to DNA damage in BCR-ABLexpressing cells. Anticancer Agents Med Chem: Nov 13, 2012 (Epub ahead of print).

28. Harry GJ and Kraft AD: Neuroinflammation and microglia: considerations and approaches for neurotoxicity assessment. Expert Opin Drug Metab Toxicol 4: 1265-1277, 2008.

29. Block ML, Zecca L and Hong JS: Microglia-mediated neurotoxicity: uncovering the molecular mechanisms. Nat Rev Neurosci 8: 57-69, 2007. 